\title{
A generalized Hyers-Ulam stability of a Pexiderized logarithmic functional equation in restricted domains
}

\author{
Jae-Young Chung
}

\section{Correspondence: jychung@kunsan. ac.kr \\ Department of Mathematics, Kunsan National University, Kunsan 573-701, Republic of Korea}

\begin{abstract}
Let $\mathbb{R}_{+}$and $B$ be the set of positive real numbers and a Banach space, respectively, $f$, $g, h: \mathbb{R}_{+} \rightarrow B$ and $\psi: \mathbb{R}_{+}^{2} \rightarrow \mathbb{R}$ be a nonnegative function of some special forms. Generalizing the stability theorem for a Jensen-type logarithmic functional equation, we prove the Hyers-Ulam stability of the Pexiderized logarithmic functional inequality

$$
\|f(x y)-g(x)-h(y)\| \leq \psi(x, y)
$$

in restricted domains of the form $\left\{(x, y): x^{k} y^{s} \geq d\right\}$ for fixed $k, s \in \mathbb{R}, d>0$. We also discuss an $L^{\infty}$-version of the Hyers-Ulam stability of the inequality. 2000 MSC: 39B22.

Keywords: logarithmic functional equation, Hyers-Ulam stability, asymptotic behavior
\end{abstract}

\section{Introduction}

The Hyers-Ulam stability problems of functional equations go back to 1940 when Ulam proposed a question concerning the approximate homomorphisms from a group to a metric group (see [1]). A partial answer was given by Hyers [2,3] under the assumption that the target space of the involved mappings is a Banach space. After the result of Hyers, Aoki [4] and Bourgin [5,6] treated with this problem, however, there were no other results on this problem until 1978 when Rassias [7] treated again with the inequality of Aoki [4]. Following the Rassias' result a great number of articles on the subject have been published concerning numerous functional equations in various directions [8-19]. Among the results, the stability problem in a restricted domain was investigated by Skof, who proved the stability problem of the Cauchy functional equation in a restricted domain [20]. Developing this result, Jung, Rassias and Rassias considered the stability problems in restricted domains for the Jensen functional equation $[21,22]$ and Jensen-type functional equations [23]. We also refer the reader to [24-29] for some interesting results on functional equations and their Hyers-Ulam stabilities in restricted conditions. In this article, generalizing the result in [8], we consider the Hyers-Ulam stability of the Pexiderized Jensen functional equation

$$
\|f(x y)-g(x)-h(y)\| \leq \psi(x, y)
$$

in the restricted domains $U_{k, s, d}=\left\{(x, y): x>0, y>0, x^{k} y^{s} \geq d\right\}$ for fixed $k, s \in \mathbb{R}$ and $d>0$, where $\psi(x, y)=\varphi(x y), \varphi(x)$ or $\varphi(y)$. Making use of the result, we prove the

(c) 2012 Chung; licensee Springer. This is an Open Access article distributed under the terms of the Creative Commons Attribution License (http://creativecommons.org/licenses/by/2.0), which permits unrestricted use, distribution, and reproduction in any medium, provided the original work is properly cited. 
asymptotic behavior of $f, g$ and $h$ satisfying

$$
\|f(x y)-g(x)-h(y)\| \rightarrow 0
$$

as $x^{k} y^{s} \rightarrow \infty$. Finally, we discuss the Hyers-Ulam stability of the inequality

$$
\|f(x y)-g(x)-h(y)\|_{L^{\infty}\left(U_{k, s, d}\right)} \leq \varepsilon
$$

and its asymptotic behavior.

\section{Stability in classical sense}

We call $L: \mathbb{R}_{+} \rightarrow B$ a logarithmic function provided that

$$
L(x y)-L(x)-L(y)=0
$$

for all $x, y>0$. Let $\varphi: \mathbb{R}_{+} \rightarrow[0, \infty)$. We assume that

$$
\Phi(x):=\sum_{k=1}^{\infty} 2^{-k}\left(\phi\left(x^{2^{k}}\right)+2 \phi\left(x^{2^{k-1}}\right)+\phi(1)\right)<\infty
$$

for all $x>0$. As a direct consequence of Aoki [4] or Bourgin [5,6], we obtain the generalized Hyers-Ulam stability for the logarithmic functional equation, viewing $\left\langle\mathbb{R}_{+}\right.$, $x>$ as a multiplicative group.

Theorem A. Suppose that $f: \mathbb{R}_{+} \rightarrow B$ satisfies

$$
\|f(x y)-f(x)-f(y)\| \leq \phi(x y)+\phi(x)+\phi(y)+\phi(1)
$$

for all $x, y>0$. Then, there exists a unique logarithmic function $L: \mathbb{R}_{+} \rightarrow B$ satisfying

$$
\|f(x)-L(x)\| \leq \Phi(x)
$$

for all $x>0$.

In this section, we first consider the logarithmic functional inequality (1.1) in the restricted domain

$$
U_{k, s, d}=\left\{(x, y): x>0, y>0, x^{k} y^{s} \geq d\right\}
$$

for fixed $k, s \in \mathbb{R}$ and $d>0$.

Theorem 2.1. Let $d>0, k, s \in \mathbb{R}, k \neq s$. Suppose that $f, g, h: \mathbb{R}_{+} \rightarrow B$ satisfy

$$
\|f(x y)-g(x)-h(y)\| \leq \phi(x y)
$$

for all $x, y \in U_{k, s, d}$. Then, there exists a unique logarithmic function $L_{1}: \mathbb{R}_{+} \rightarrow B$ such that

$$
\left\|f(x)-L_{1}(x)-f(1)\right\| \leq \Phi(x)
$$

for all $x \in \mathbb{R}_{+}$.

Proof. For given $x, y \in \mathbb{R}_{+}$, choosing a $z>0$ such that $x^{k} y^{s} z^{s-k} \geq d, x^{k} z^{s-k} \geq d, y^{s} z^{s-k} \geq d$ and $z^{s-k} \geq d$, we have 


$$
\begin{aligned}
\|f(x y)-f(x)-f(y)+f(1)\| \leq & \left\|f(x y)-g\left(x z^{-1}\right)-h(y z)\right\| \\
& +\left\|-f(x)+g\left(x z^{-1}\right)+h(z)\right\| \\
& +\left\|-f(y)+g\left(z^{-1}\right)+h(y z)\right\| \\
& +\left\|f(1)-g\left(z^{-1}\right)-h(z)\right\| \\
\leq & \phi(x y)+\phi(x)+\phi(y)+\phi(1) .
\end{aligned}
$$

Now, by Theorem A, we get the result.

Corollary 2.2. Let $\epsilon, d>0, k, s \in \mathbb{R}, k \neq s$. Suppose that $f, g, h: \mathbb{R}_{+} \rightarrow B$ satisfy

$$
\|f(x y)-g(x)-h(y)\| \leq \varepsilon
$$

for all $x, y \in U_{k, s, d}$. Then, there exists a unique logarithmic function $L_{1}: \mathbb{R}_{+} \rightarrow B$ such that

$$
\left\|f(x)-L_{1}(x)-f(1)\right\| \leq 4 \varepsilon
$$

for all $x \in \mathbb{R}_{+}$.

Remark 2.1. Note that the Corollary 2.2 fails if $k=s$. Indeed, let $L: \mathbb{R}_{+} \rightarrow B$ be a nonzero logarithmic function. Define $g(x)=h(x)=L(x)$ for all $x>0$ and

$$
f(x)= \begin{cases}L(x), & x \geq d^{1 / s} \\ 0, & 0<x<d^{1 / s} .\end{cases}
$$

Then, it is easy to see that the inequality (2.4) holds for all $x, y>0$, with $x y \geq d^{1 / s}$. Assume that there exists a logarithmic function $L_{1}$ satisfying (2.5). Then, we have

$$
|| L_{1}(x)|| \leq|f(1)|+4 \varepsilon=4 \varepsilon
$$

for all $0<x<d^{1 / s}$. The inequality (2.6) implies $L_{1}=0$. Indeed, if $L_{1}\left(x_{0}\right) \neq 0$ for some $x_{0}>0$, then we have $L_{1}\left(1 / x_{0}\right)=-L_{1}\left(x_{0}\right) \neq 0$. Thus, we may assume that $0<x_{0}<1$. Now, we encounter the contradiction

$$
\left|n L_{1}\left(x_{0}\right)\right|=\left|L_{1}\left(x_{0}^{n}\right)\right| \leq 4 \varepsilon
$$

for all large integers $n$. Thus, $L_{1}=0$ and the inequality (2.5) implies

$$
\|L(x)\| \leq 4 \varepsilon
$$

for all $x \geq d^{1 / s}$. Similarly, using (2.7), we can show that $L=0$, which contracts to the choice of $L$.

As a direct consequence of Corollary 2.2, we have the following.

Corollary 2.3. [8] Let $p, q, P, Q$ be nonzero real numbers and $\varepsilon, d>0, k, s \in \mathbb{R}, \frac{k}{p} \neq \frac{s}{q}$. Suppose that $f: \mathbb{R}_{+} \rightarrow B$ satisfies

$$
\left\|f\left(x^{p} \gamma^{q}\right)-P f(x)-Q f(y)\right\| \leq \varepsilon
$$

for all $x, y \in U_{k, s, d}$. Then, there exists a unique logarithmic function $L: \mathbb{R}_{+} \rightarrow B$ such that

$$
\|f(x)-L(x)-f(1)\| \leq 4 \varepsilon
$$

for all $x \in \mathbb{R}_{+}$. 
Proof. Replacing $x$ by $x^{\frac{1}{p}}, y$ by $y^{\frac{1}{q}}$ in (2.8), we have

$$
\left\|f(x y)-P f\left(x^{\frac{1}{p}}\right)-Q f\left(y^{\frac{1}{q}}\right)\right\| \leq \varepsilon
$$

for all $x, y>0$, with $x^{\frac{k}{p}} y^{\frac{s}{q}} \geq d$. Letting $g(x)=\operatorname{Pf}\left(x^{\frac{1}{p}}\right), h(y)=Q f\left(y^{\frac{1}{q}}\right)$, applying Corollary 2.2 and letting $L(x)=L_{1}(x)$, we get the result.

Theorem 2.4. Let $d>0, s \neq 0$. Suppose that $f, g, h: \mathbb{R}_{+} \rightarrow B$ satisfy

$$
\|f(x y)-g(x)-h(y)\| \leq \phi(x)
$$

for all $x, y \in U_{k, s, d}$. Then, there exists a unique logarithmic function $L_{2}: \mathbb{R}_{+} \rightarrow B$ such that

$$
\left\|g(x)-L_{2}(x)-g(1)\right\| \leq \Phi(x)
$$

for all $x \in \mathbb{R}_{+}$.

Proof. For given $x, y \in \mathbb{R}_{+}$, choosing a $z>0$ such that $x^{k} y^{k} z^{s} \geq d, x^{k} y^{s} z^{s} \geq d, y^{k} z^{s} \geq d$ and $y^{s} z^{s} \geq d$, we have

$$
\begin{aligned}
\|g(x y)-g(x)-g(y)+g(1)\| \leq & \|-f(x y z)+g(x y)+h(z)\| \\
& +\|f(x y z)-g(x)-h(y z)\| \\
& +\|f(y z)-g(y)-h(z)\| \\
& +\|-f(y z)+g(1)+h(y z)\| \\
\leq & \phi(x y)+\phi(x)+\phi(y)+\phi(1) .
\end{aligned}
$$

Now, by Theorem A, we get the result.

Corollary 2.5. Let $\epsilon, d>0, s \neq 0$. Suppose that $f, g, h: \mathbb{R}_{+} \rightarrow B$ satisfy

$$
\|f(x y)-g(x)-h(y)\| \leq \varepsilon
$$

for all $x, y \in U_{k, s, d}$. Then, there exists a unique logarithmic function $L_{2}: \mathbb{R}_{+} \rightarrow B$ such that

$$
\left\|g(x)-L_{2}(x)-g(1)\right\| \leq 4 \varepsilon
$$

for all $x \in \mathbb{R}_{+}$.

Remark 2.2. Similarly as in Corollary 2.2, the above result fails if $s=0$. Let $L: \mathbb{R}_{+} \rightarrow$ $B$ be a nonzero logarithmic function. Define $f(x)=h(x)=L(x)$ for all $x>0$ and

$$
g(x)= \begin{cases}L(x), & x \geq d^{1 / k} \\ 0, & 0<x<d^{1 / s}\end{cases}
$$

Then, the inequality (2.13) holds for all $x, y>0$, with $x^{k} \geq d$ but (2.14) does not hold for any logarithmic function $L_{2}$.

As a direct consequence of Corollary 2.5, we have the following.

Corollary 2.6. [8] Let $p, q, P, Q$ be nonzero real numbers and $\epsilon, d>0, k, s \in \mathbb{R}$ with $s$ $\neq 0$. Suppose that $f: \mathbb{R}_{+} \rightarrow B$ satisfies

$$
\left\|f\left(x^{p} \gamma^{q}\right)-P f(x)-Q f(y)\right\| \leq \varepsilon
$$

for all $x, y \in U_{k, s, d}$. Then, there exists a unique logarithmic function $L: \mathbb{R}_{+} \rightarrow B$ such that 


$$
\|f(x)-L(x)-f(1)\| \leq \frac{4 \varepsilon}{|P|}
$$

for all $x \in \mathbb{R}_{+}$.

Proof. Replacing $x$ by $x^{\frac{1}{p}}, y$ by $y^{\frac{1}{q}}$ in (2.15), we have

$$
\left\|f(x y)-P f\left(x^{\frac{1}{p}}\right)-Q f\left(y^{\frac{1}{q}}\right)\right\| \leq \varepsilon
$$

for all $x, y>0$, with $x^{\frac{k}{p}} y^{\frac{s}{q}} \geq d$. Letting $g(x)=P f\left(x^{\frac{1}{p}}\right), h(y)=Q f\left(y^{\frac{1}{q}}\right)$, applying Corollary 2.5 and dividing the result by $|P|$, we get the result with $L(x)=\frac{1}{P} L_{2}\left(x^{p}\right)$.

Theorem 2.7. Let $d>0, k \neq 0$. Suppose that $f, g, h: \mathbb{R}_{+} \rightarrow B$ satisfy

$$
\|f(x y)-g(x)-h(y)\| \leq \phi(y)
$$

for all $x, y \in U_{k, s, d}$. Then, there exists a unique logarithmic function $L_{3}: \mathbb{R}_{+} \rightarrow B$ such that

$$
\left\|h(x)-L_{3}(x)-h(1)\right\| \leq \Phi(x)
$$

for all $x \in \mathbb{R}_{+}$.

Proof. For given $x, y \in \mathbb{R}_{+}$, choosing a $z>0$ such that $x^{s} y^{s} z^{k} \geq d, x^{k} y^{s} z^{k} \geq d, x^{s} z^{k} \geq d$ and $x^{k} z^{k} \geq d$, we have

$$
\begin{aligned}
\|h(x y)-h(x)-h(y)+h(1)\| \leq & \|-f(x y z)+g(z)+h(x y)\| \\
& +\|f(x y z)-g(x z)-h(y)\| \\
& +\|f(z x)-g(z)-h(x)\| \\
& +\|-f(x z)+g(x z)+h(1)\| \\
\leq & \phi(x y)+\phi(x)+\phi(y)+\phi(1) .
\end{aligned}
$$

Now, by Theorem A, we get the result.

Corollary 2.8. Let $\epsilon, d>0, k \neq 0$. Suppose that $f, g, h: \mathbb{R}_{+} \rightarrow B$ satisfy

$$
\|f(x y)-g(x)-h(y)\| \leq \varepsilon
$$

for all $x, y \in U_{k, s, d}$. Then, there exists a unique logarithmic function $L_{3}: \mathbb{R}_{+} \rightarrow B$ such that

$$
\left\|h(x)-L_{3}(x)-h(1)\right\| \leq 4 \varepsilon
$$

for all $x \in \mathbb{R}_{+}$.

Remark 2.3. Similarly, as in Remark 2.2, we can show that the above result fails if $k$ $=0$. Also, as a direct consequence of the result, we have the following.

Corollary 2.9. [8] Let $p, q, P, Q$ be nonzero real numbers and $\epsilon, d>0, k, s \in \mathbb{R}$ with $k$ $\neq 0$. Suppose that $f: \mathbb{R}_{+} \rightarrow B$ satisfies

$$
\left\|f\left(x^{p} \gamma^{q}\right)-P f(x)-Q f(y)\right\| \leq \varepsilon
$$

for all $x, y \in U_{k, s, d}$. Then, there exists a unique logarithmic function $L: \mathbb{R}_{+} \rightarrow B$ such that 


$$
|| f(x)-L(x)-f(1)|| \leq \frac{4 \varepsilon}{|Q|}
$$

for all $x \in \mathbb{R}_{+}$.

Theorem 2.10. Let $\epsilon, d>0, k, s \neq 0, k \neq s$. Suppose that $f, g, h: \mathbb{R}_{+} \rightarrow B$ satisfy

$$
\|f(x y)-g(x)-h(y)\| \leq \varepsilon
$$

for all $x, y \in U_{k, s, d}$. Then, there exists a unique logarithmic function $L: \mathbb{R}_{+} \rightarrow B$ such that

$$
\begin{aligned}
& \|f(x)-L(x)-f(1)\| \leq 4 \varepsilon \\
& \|g(x)-L(x)-g(1)\| \leq 4 \varepsilon \\
& \|h(x)-L(x)-h(1)\| \leq 4 \varepsilon
\end{aligned}
$$

for all $x \in \mathbb{R}_{+}$.

Proof. In view of Corollaries 2.2, 2.5 and 2.8, it suffices to prove that $L_{1}=L_{2}=L_{3}$. For given $x, y>0$, choose a $z>0$ such that $x^{k} y^{s} z^{s-k} \geq d, z^{s-k} \geq d$. Then, in view of (2.24), we have

$$
\begin{aligned}
& \left\|f(x y)-g\left(x z^{-1}\right)-h(y z)\right\| \leq \varepsilon, \\
& \left\|-f(1)+g\left(z^{-1}\right)+h(z)\right\| \leq \varepsilon .
\end{aligned}
$$

Using the inequalities (2.10) and (2.15), we have

$$
\begin{aligned}
& \left\|g\left(x z^{-1}\right)-g(x)-g\left(z^{-1}\right)+g(1)\right\| \leq 4 \varepsilon, \\
& \|h(y z)-h(z)-h(y)+h(1)\| \leq 4 \varepsilon
\end{aligned}
$$

for all $x, y, z>0$. From (2.25)-(2.28), using the triangle inequality, we have

$$
\|f(x y)-g(x)-h(y)-f(1)+g(1)+h(1)\| \leq 10 \varepsilon
$$

for all $x, y>0$. From the inequalities (2.5), (2.14), (2.21), (2.29) using the triangle inequality, we have

$$
\left\|L_{1}(x y)-L_{2}(x)-L_{3}(y)\right\| \leq 22 \varepsilon .
$$

Putting $y=1$ and $x=1$ in (2.30) separately, and using the fact that for all $x>0, n \in$ $\mathbb{N}, L_{j}\left(x^{n}\right)=n L_{j}(x), j=1,2,3$, we can show that $L_{1}=L_{2}$ and $L_{1}=L_{3}$. This completes the proof.

As a direct consequence of Theorem 2.10, we have the following.

Corollary 2.11. [8] Let $p, q, P, Q$ be nonzero real numbers and $\epsilon, d>0, k, s \in \mathbb{R}$ with $k \neq 0, s \neq 0$ and $k \neq s$. Suppose that $f: \mathbb{R}_{+} \rightarrow B$ satisfies

$$
\left\|f\left(x^{p} y^{q}\right)-P f(x)-Q f(y)\right\| \leq \varepsilon
$$

for all $x, y \in U_{k, s, d}$. Then, there exists a unique logarithmic function $L: \mathbb{R}_{+} \rightarrow B$ such that

$$
\|f(x)-L(x)-f(1)\| \leq \min \left\{4 \varepsilon, \frac{4 \varepsilon}{|P|}, \frac{4 \varepsilon}{|Q|}\right\}
$$


for all $x \in \mathbb{R}_{+}$.

\section{Asymptotic behaviors}

In this section, we consider asymptotic behaviors of $f, g$, $h$ satisfying (1.2).

Theorem 3.1. Let $k, s \in \mathbb{R}, k \neq s$. Suppose that $f, g, h: \mathbb{R}_{+} \rightarrow B$ satisfy the asymptotic condition

$$
\|f(x y)-g(x)-h(y)\| \rightarrow 0
$$

as $x^{k} y^{s} \rightarrow \infty$. Then, there exists a unique logarithmic function $L: \mathbb{R}_{+} \rightarrow B$ such that

$$
f(x)=L(x)+f(1)
$$

for all $x \in \mathbb{R}_{+}$.

Proof. By the condition (3.1), for each $n \in \mathbb{N}$, there exists $d_{n}>0$ such that

$$
\|f(x y)-g(x)-h(y)\| \leq \frac{1}{n}
$$

for all $x, y>0$, with $x^{k} y^{s} \geq d_{n}$. By Corollary 2.2, there exists a unique logarithmic function $L_{n}: \mathbb{R}_{+} \rightarrow B$ such that

$$
\left\|f(x)-L_{n}(x)-f(1)\right\| \leq \frac{4}{n}
$$

for all $x \in \mathbb{R}_{+}$. Replacing $n$ by $m$ in (3.4) and using the triangle inequality we have

$$
\left\|L_{n}(x)-L_{m}(x)\right\| \leq \frac{4}{n}+\frac{4}{m} \leq 8
$$

for all $x \in \mathbb{R}_{+}$. Now, for all $x>0$ and all rational numbers $r>0$, we have

$$
\left\|L_{n}(x)-L_{m}(x)\right\|=\frac{1}{r}\left\|L_{n}\left(x^{r}\right)-L_{m}\left(x^{r}\right)\right\| \leq \frac{8}{r} .
$$

Letting $r \rightarrow \infty$ in (3.6), we have $L_{n}=L_{m}$. Letting $n \rightarrow \infty$ in (3.4), we get the result. Using Corollary 2.5, we obtain the following.

Theorem 3.2. Let $s \neq 0$. Suppose that $f, g, h: \mathbb{R}_{+} \rightarrow B$ satisfy the asymptotic condition

$$
\|f(x y)-g(x)-h(y)\| \rightarrow 0
$$

as $x^{k} y^{s} \rightarrow \infty$. Then, there exists a unique logarithmic function $L: \mathbb{R}_{+} \rightarrow B$ such that

$$
g(x)=L(x)+g(1)
$$

for all $x \in \mathbb{R}_{+}$.

Using Corollary 2.8, we obtain the following.

Theorem 3.3. Let $k \neq 0$. Suppose that $f, g, h: \mathbb{R}_{+} \rightarrow B$ satisfies the asymptotic condition

$$
\|f(x y)-g(x)-h(y)\| \rightarrow 0
$$

as $x^{k} y^{s} \rightarrow \infty$. Then, there exists a unique logarithmic function $L: \mathbb{R}_{+} \rightarrow B$ such that

$$
h(x)=L(x)+h(1)
$$

for all $x \in \mathbb{R}_{+}$. 
Theorem 3.4. Let $k, s \neq 0$ and $k \neq s$. Suppose that $f, g, h: \mathbb{R}_{+} \rightarrow B$ satisfy the asymptotic condition

$$
\|f(x y)-g(x)-h(y)\| \rightarrow 0
$$

as $x^{k} y^{s} \rightarrow \infty$. Then, there exists a unique logarithmic function $L: \mathbb{R}_{+} \rightarrow B$ and $c_{1}, c_{2}$ $\in B$ such that

$$
\begin{aligned}
& f(x)=L(x)+c_{1}+c_{2} \\
& g(x)=L(x)+c_{1} \\
& h(x)=L(x)+c_{2}
\end{aligned}
$$

for all $x \in \mathbb{R}_{+}$.

Proof. By the condition (3.11), for each $n \in \mathbb{N}$, there exists $d_{n}>0$ such that

$$
\|f(x y)-g(x)-h(y)\| \leq \frac{1}{n}
$$

for all $x, y>0$, with $x^{k} y^{s} \geq d_{n}$. By Theorem 2.10, there exists a unique logarithmic function $L_{n}: \mathbb{R}_{+} \rightarrow B$ such that

$$
\begin{aligned}
& \left\|f(x)-L_{n}(x)-f(1)\right\| \leq \frac{4}{n}, \\
& \left\|g(x)-L_{n}(x)-g(1)\right\| \leq \frac{4}{n^{\prime}} \\
& \left\|h(x)-L_{n}(x)-h(1)\right\| \leq \frac{4}{n}
\end{aligned}
$$

for all $x \in \mathbb{R}_{+}$. Similarly, as in the proof of Theorem 3.1, we have $L_{n}=L_{m}$ for all $n$, $m \in \mathbb{N}$. Letting $n \rightarrow \infty$ in (3.13)-(3.15), and using (3.11), we get the result.

\section{Stability in $L^{\infty}$-sense and its asymptotic behavior}

Let $f, g, h$ be locally integrable functions on $\mathbb{R}^{+}$. In this section, we consider the $L^{\infty}$-version of Hyers-Ulam stability of the inequality

$$
\|f(x y)-g(x)-h(y)\|_{L^{\infty}\left(U_{k, s, d}\right)} \leq \varepsilon,
$$

where $k \neq 0, s \neq 0, k \neq s, d>0$ are fixed and $U_{k, s, d}=\left\{(x, y): x^{k} y^{s} \geq d\right\}$. Let $\omega$ on $\mathbb{C}$ be a nonnegative infinitely differentiable function satisfying the conditions

$$
\operatorname{supp} \omega \subset\{x:|x| \leq 1\}
$$

and

$$
\int \omega(x) d x=1
$$

Let $\omega_{t}(x):=t^{-1} \omega(x / t), t>0$ and $f$ be a locally integrable function. Then, for each $t>0$, $f^{*} \omega_{t}(x)=\int f(y) \omega_{t}(x-y) d y$ is a smooth function of $x \in \mathbb{C}$ and $f^{*} \omega_{t}(x) \rightarrow f(x)$ for almost every $x \in \mathbb{C}$ as $t \rightarrow 0^{+}$. Now, we are in a position to prove the Hyers-Ulam stability of the inequality (3.1). 
Theorem 4.1. Let $f, g, h$ be locally integrable functions satisfying (3.1). Then, there exist $c_{1}, c_{2}, c_{3}, a \in \mathbb{C}$ such that

$$
\begin{aligned}
& \left\|f(x)-c_{1}-a \ln x\right\|_{L^{\infty}\left(\mathbb{R}_{+}\right)} \leq 4 \varepsilon, \\
& \left\|g(x)-c_{2}-a \ln x\right\|_{L^{\infty}\left(\mathbb{R}_{+}\right)} \leq 4 \varepsilon, \\
& \left\|h(x)-c_{3}-a \ln x\right\|_{L^{\infty}\left(\mathbb{R}_{+}\right)} \leq 4 \varepsilon .
\end{aligned}
$$

Proof. Using the change of variables $x$ by $2^{x}$ and $y$ by $2^{y}$ in (4.1), we have

$$
\left\|f\left(2^{x+y}\right)-g\left(2^{x}\right)-h\left(2^{y}\right)\right\|_{L^{\infty}\left(U_{d}\right)} \leq \varepsilon,
$$

where $U_{d}=\left\{(x, y): k x+s y \geq \log _{2}^{d}:=d_{1}\right\}$. Now, let

$$
F(x)=f\left(2^{x}\right), \quad G(x)=g\left(2^{x}\right), \quad H(x)=h\left(2^{x}\right) .
$$

Then, we have

$$
\|F(x+y)-G(x)-H(y)\|_{L^{\infty}\left(U_{d}\right)} \leq \varepsilon .
$$

Convolving $\omega_{t}(x) \omega_{s}(y)$ in (4.4) as in the proof of [8, Theorem 3.1], we have

$$
\left|F * \omega_{t} * \omega_{s}(x+y)-G * \omega_{t}(x)-H * \omega_{s}(y)\right| \leq \varepsilon
$$

holds for all $k x+s y \geq d_{2}:=d_{1}+\sqrt{k^{2}+s^{2}}$ and $0<t<1,0<s<1$. Using the same method as in [9, Theorem 4.3], we get the result.

Now, we discuss an asymptotic behavior of the inequality (4.1).

Theorem 4.2. Let $f, g, h: \mathbb{R}_{+} \rightarrow \mathbb{C}, j=1,2,3$, be locally integrable functions satisfying

$$
\|f(x y)-g(x)-h(y)\|_{L^{\infty}\left(U_{k, s, d}\right)} \rightarrow 0
$$

as $d \rightarrow \infty$. Then, there exist $a, c_{1}, c_{2}, c_{3} \in \mathbb{C}$ such that

$$
\begin{aligned}
& \left\|f(x)-c_{1}-a \ln x\right\|_{L^{\infty}\left(\mathbb{R}_{+}\right)}=0, \\
& \left\|g(x)-c_{2}-a \ln x\right\|_{L^{\infty}\left(\mathbb{R}_{+}\right)}=0, \\
& \left\|h(x)-c_{3}-a \ln x\right\|_{L^{\infty}\left(\mathbb{R}_{+}\right)}=0 .
\end{aligned}
$$

Proof. By the condition (4.6), for any positive integer $n$, there exists $d_{n}>0$ such that

$$
\|f(x y)-g(x)-h(y)\|_{L^{\infty}\left(U_{k, s, d_{n}}\right)} \leq \frac{1}{n}
$$

for all $x, y \in U_{k, s, d_{n}}$. Now, by Theorem 4.1, there exist $a, c_{1}, c_{2}, c_{3} \in \mathbb{C}$ (which are independent of $n$ ) such that

$$
\begin{aligned}
& \left\|f(x)-c_{1}-a \ln x\right\|_{L^{\infty}\left(\mathbb{R}_{+}\right)} \leq \frac{4}{n^{\prime}} \\
& \| g(x)-c_{2}-a \ln x--_{L^{\infty}\left(\mathbb{R}_{+}\right)} \leq \frac{4}{n^{\prime}} \\
& \left\|h(x)-c_{3}-a \ln x\right\|_{L^{\infty}\left(\mathbb{R}_{+}\right)} \leq \frac{4}{n} .
\end{aligned}
$$




\section{Acknowledgements}

This study was supported by the Basic Science Research Program through the National Research Foundation of Korea (NRF) funded by the Ministry of Education, Science and Technology (MEST) (No. 2010-0016963).

\section{Competing interests}

The author declares that they have no competing interests.

Received: 9 May 2011 Accepted: 19 January 2012 Published: 19 January 2012

\section{References}

1. Ulam, SM: A Collection of Mathematical Problems. Interscience Publishers, New York (1960)

2. Hyers, DH: On the stability of the linear functional equations. Proc Natl Acad Sci USA. 27, 222-224 (1941). doi:10.1073/ pnas.27.4.222

3. Hyers, DH, Isac, G, Rassias, ThM: Stability of Functional Equations in Several Variables. Birkhauser, Basel (1998)

4. Aoki, T: On the stability of the linear transformation in Banach spaces. J Math Soc Japan. 2, $64-66$ (1950). doi:10.2969/ jmsj/00210064

5. Bourgin, DG: Class of transformations and bordering transformations. Bull Amer Math Soc. 57, 223-237 (1951). doi:10.1090/50002-9904-1951-09511-7

6. Bourgin, DG: Multiplicative transformations. Proc Natl Acad Sci USA. 36, 564-570 (1950). doi:10.1073/pnas.36.10.564

7. Rassias, ThM: On the stability of linear mapping in Banach spaces. Proc Amer Math Soc. 72, 297-300 (1978). doi:10.1090/S0002-9939-1978-0507327-1

8. Chung, J: Stability of a Jensen type logarithmic functional equation on restricted domains and its asymptotic behaviors. Adv Diff Equ 2010, 13 (2010). Article ID 432796

9. Chung, J: A distributional version of functional equations and their stabilities. Nonlinear Anal. 62, 1037-1051 (2005). doi:10.1016/j.na.2005.04.016

10. Czerwik, S: Stability of Functional Equations of Ulam-Hyers-Rassias Type. Hadronic Press, Inc., Palm Harbor (2003)

11. Czerwik, S: Functional Equations and Inequalities in Several Variables. World Scientific Publ. Co., Singapore (2002)

12. Forti, GL: The stability of homomorphisms and amenability with applications to functional equations. Abh Math Sem Univ Hamburg. 57, 215-226 (1987). doi:10.1007/BF02941612

13. Jun, KW, Kim, HM: Stability problem for Jensen-type functional equations of cubic mappings. Acta Math Sin Engl Ser. 22(6), 1781-1788 (2006). doi:10.1007/s10114-005-0736-9

14. Kim, GH, Lee, YH: Boundedness of approximate trigonometric functional equations. Appl Math Lett. 31, 439-443 (2009)

15. Kannappan, Pl: Functional Equations and Inequalities with Applications. Springer, New York (2009)

16. Rassias, JM: On the Ulam stability of mixed type mappings on restricted domains. J Math Anal Appl. 276, 747-762 (2002). doi:10.1016/50022-247X(02)00439-0

17. Rassias, JM: On approximation of approximately linear mappings by linear mappings. J Funct Anal. 46, 126-130 (1982). doi:10.1016/0022-1236(82)90048-9

18. Rassias, ThM: On the stability of functional equations and a problem of Ulam. Acta Appl Math. 62(1), 23-130 (2000). doi:10.1023/A:1006499223572

19. Hyers, DH, Rassias, ThM: Approximate homomorphisms. Aequationes Math. 44, 125-153 (1992). doi:10.1007/BF01830975

20. Skof, F: Sull'approssimazione delle applicazioni localmente $\omega$-additive. Atii Accad Sci Torino Cl Sci Fis Mat Natur. 117, 377-389 (1983)

21. Jung, SM: Hyers-Ulam-Rassias Stability of Functional Equations in Nonlinear Analysis. Springer, New York (2011)

22. Jung, SM: Hyers-Ulam stability of Jensen's equation and its application. Proc Amer Math Soc. 126, 3137-3143 (1998). doi:10.1090/50002-9939-98-04680-2

23. Rassias, JM, Rassias, MJ: On the Ulam stability of Jensen and Jensen type mappings on restricted domains. J Math Anal Appl. 281, 516-524 (2003). doi:10.1016/50022-247X(03)00136-7

24. Batko, B: Stability of an alternative functional equation. J Math Anal Appl. 339, 303-311 (2008). doi:10.1016/j. jmaa.2007.07.001

25. Batko, B: On approximation of approximate solutions of Dhombres' equation. J Math Anal Appl. 340, 424-432 (2008). doi:10.1016/j.jmaa.2007.08.009

26. Brzdek, J: On a method of proving the Hyers-Ulam stability of functional equations on restricted domains. Austral J Math Anal Appl. 6, 1-10 (2009)

27. Brzdek, J: On stability of a family of functional equations. Acta Math Hungarica. 128, 139-149 (2010). doi:10.1007/ s10474-010-9169-8

28. Sikorska, J: On a Pexiderized conditional exponential functional equation. Acta Math Hun-garica. 125, 287-299 (2009). doi:10.1007/s10474-009-9019-8

29. Sikorska, J: Exponential functional equation on spheres. Appl Math Lett. 23, 156-160 (2010). doi:10.1016/j. aml.2009.09.004 